\title{
Weighted self-avoiding walks
}

\author{
Geoffrey R. Grimmett ${ }^{1,2}$. Zhongyang $\mathrm{Li}^{3}$
}

Received: 17 April 2018 / Accepted: 6 June 2019 / Published online: 18 June 2019

(c) The Author(s) 2019

\begin{abstract}
We study the connective constants of weighted self-avoiding walks (SAWs) on infinite graphs and groups. The main focus is upon weighted SAWs on finitely generated, virtually indicable groups. Such groups possess so-called height functions, and this permits the study of SAWs with the special property of being bridges. The group structure is relevant in the interaction between the height function and the weight function. The main difficulties arise when the support of the weight function is unbounded, since the corresponding graph is no longer locally finite. There are two principal results, of which the first is a condition under which the weighted connective constant and the weighted bridge constant are equal. When the weight function has unbounded support, we work with a generalized notion of the 'length' of a walk, which is subject to a certain condition. In the second main result, the above equality is used to prove a continuity theorem for connective constants on the space of weight functions endowed with a suitable distance function.
\end{abstract}

Keywords Self-avoiding walk · Weight function · Connective constant · Bridge constant · Virtually indicable group · Cayley graph · Transitive graph

Mathematics Subject Classification 05C30 $\cdot$ 20F65 $\cdot 82 \mathrm{~B} 20$

Geoffrey R. Grimmett

g.r.grimmett@ statslab.cam.ac.uk

http://www.statslab.cam.ac.uk/ grg/

Zhongyang Li

zhongyang.li@uconn.edu

http://www.math.uconn.edu/ zhongyang/

1 Statistical Laboratory, Centre for Mathematical Sciences, Cambridge University, Wilberforce Road, Cambridge CB3 OWB, UK

2 School of Mathematics and Statistics, The University of Melbourne, Parkville, VIC 3010, Australia

3 Department of Mathematics, University of Connecticut, Storrs, CT 06269-3009, USA 


\section{Introduction}

The counting of self-avoiding walks (SAWs) is extended here to the study of weighted SAWs. Each edge is assigned a weight, and the weight of a SAW is defined as the product of the weights of its edges. We study certain properties of the exponential growth rate $\mu$ (known as the connective constant) in terms of the weight function $\phi$, including its continuity on the space of weight functions.

The theory largely follows the now established route when the underlying graph $G$ is locally finite. New problems emerge when $G$ is not locally finite, and these are explored in the situation in which $G$ is the complete graph on an infinite group $\Gamma$, with weight function $\phi$ defined on $\Gamma$.

One of the main technical steps in the current work is the proof, subject to certain conditions, of the equality of the weighted connective constant and the weighted bridge constant. This was proved in [12, Thm 4.3] for the unweighted constants on any connected, infinite, quasi-transitive, locally finite, simple graph possessing a unimodular graph height function. ${ }^{1}$ This result is extended here to weighted SAWs on finitely generated, virtually indicable groups (see Sect. 3 and $[17,18]$ for references on virtual indicability).

As explained in [11,12], a key assumption for the definition and study of bridge SAWs on an unweighted graph is the existence of a so-called graph height function $h$. A 'bridge' is a SAW $\pi$ for which there exists an interval $[a, b]$ such that the vertices of $\pi$ have heights lying in $[a, b]$, and the initial (respectively, final) vertex of $\pi$ has height $a$ (respectively, $b$ ). The 'bridge constant' $\beta$ is the exponential growth rate of the number of bridges with length $n$ and fixed initial vertex. We note that some Cayley graphs (including those of virtually indicable groups) possess graph height functions, and some do not (see [9]).

Let $\phi$ be a non-negative weight function on the edge set of a graph, and assume $\phi$ is invariant under a certain class of graph automorphisms. The weight of a SAW $\pi$ is defined to be the product of the weights of the edges of $\pi$. By replacing the number of SAWs by their aggregate weight in the above, we may define the connective constant and the bridge constant of this weighted system of SAWs. The question arises of whether these two numbers are equal for a given graph $G$, graph height function $(h, \mathcal{H})$, and weight function $\phi$. Our proof of the equality of bridge and connective constants, given in Sects. 3 and 6, hinges on a combinatorial fact (due to Hardy and Ramanujan, [16] (see Remark 3.11)) whose application here imposes a condition on the pair $(\phi, h)$. This condition generally fails in the non-locally-finite case, but holds if the usual combinatorial definition of the length of a SAW (that is, the number of its edges) is replaced by a generalized length function $\ell$ satisfying a certain property $\Pi(\ell, h)$, stated in (3.9). In the locally finite case, the usual graph-distance function invariably satisfies $\Pi(\ell, h)$.

Certain new problems arise when working with a general length function $\ell$ other than the usual graph distance, but the reward includes the above desired equality,

\footnotetext{
1 Since the current paper was written, Lindorfer [21] has obtained a corresponding statement without the assumption of unimodularity.
} 
and also the continuity of the weighted connective constant on the space of weight functions with a suitable distance function.

Here is an informal summary of our principal results, presented in the context of weighted walks on finitely generated groups. The reader is referred to Sects. 2-4 (and in particular, Theorems 3.10 and 4.1) for formal definitions and statements.

Theorem 1.1 Let $\Gamma$ be an infinite, finitely generated, virtually indicable group, and let $\phi: \Gamma \rightarrow[0, \infty)$ be a summable, symmetric weight function that spans $\Gamma$. If (3.9) holds, then

(a) the connective and bridge constants are equal,

(b) the connective constant is a continuous function on the space $\Phi$ of such weight functions endowed with a suitable distance function.

Similar results are given for locally finite quasi-transitive graphs (see Theorems 2.5 and 2.7).

The principal results of the paper concern weighted SAWs on certain groups including, for example, all finitely generated, elementary amenable groups. The defining property of the groups under study is that they possess so-called strong graph height functions (see [10-12] and especially [9]); this is shown in Theorem 3.2 to be essentially equivalent to assuming the groups to be virtually indicable. The algebraic structure of a group $\Gamma$ plays its role through the interaction between the height function $h$, the length function $\ell$, and the weight function $\phi$.

When the support of the weight function $\phi$ is bounded, the results of this paper are fairly straightforward extensions of the unweighted case (see Sect. 2). The situation is significantly more complicated when $\phi$ has unbounded support, as in Sect. 3. Single steps of a SAW may have unbounded length in the usual graph metric, and thus, this constitutes a 'long-range' model, in the language of statistical mechanics. Longrange models have been studied in numerous contexts including percolation and the Ising model (see, for example, [1,4] and the references therein). We are unaware of applications of our SAW results to other long-range models, and indeed the principal complication in the current work, namely the restriction to length functions satisfying (3.9), does not appear to have a parallel in other systems.

Weighted SAWs have featured in earlier work of others in a variety of contexts. Randomly weighted SAWs on grids and trees have been studied by Lacoin $[19,20]$ and Chino and Sakai [2,3]. The work of Lacoin is directed at counting SAWs inside the infinite cluster of a supercritical percolation process, so that the effective weight function takes the values 0 and 1 . A type of weighted SAW on the square grid $\mathbb{Z}^{2}$ has been considered by Glazman and Manolescu [6,7], who show that the ensuing two-point function is independent of the weights so long as they conform to the corresponding Yang-Baxter equation. This may be viewed as a partial extension of results of Duminil-Copin and Smirnov [5] concerning SAWs on the triangular lattice.

Here is a summary of the contents of the article. The asymptotics of weighted self-avoiding walks on quasi-transitive, locally finite graphs are considered in Sect. 2. The relevance of graph height functions is explained in the context of bridges, and the equality of connective and bridge constants is proved for graphs possessing unimodular graph height functions. In this case, the connective constant is continuous on the space 
of weight functions with the supremum norm. Proofs are either short or even omitted, since only limited novelty is required beyond [12].

Weighted walks on a class of infinite countable groups are the subject of Sects. 3 and 4, namely, on the class of virtually indicable groups. A generalized notion of the length of a SAW is introduced, and a condition is established under which the bridge constant equals the connective constant. The connective constant is shown to be continuous in the weight and length functions. Proofs are largely deferred to Sects. 5-7.

We write $\mathbb{R}$ for the reals, $\mathbb{Z}$ for the integers, and $\mathbb{N}$ for the natural numbers.

\section{Weighted walks and bridges on locally finite graphs}

In this section, we consider weighted SAWs on locally finite graphs. The length of a walk is its conventional graph length, that is, the number of its edges.

Let $\mathcal{G}$ be the set of connected, infinite, quasi-transitive, locally finite, simple, rooted graphs, with root labelled $\mathbf{1}$. Let $G=(V, E) \in \mathcal{G}$. We weight the edges of $G$ via a function $\phi: E \rightarrow(0, \infty)$. For $\mathcal{H} \leq \operatorname{Aut}(G)$, the function $\phi$ is called $\mathcal{H}$-invariant if

$$
\phi(\langle\alpha u, \alpha v\rangle)=\phi(\langle u, v\rangle), \quad\langle u, v\rangle \in E, \alpha \in \mathcal{H} .
$$

We write

$$
\phi_{\mathrm{inf}}=\inf \{\phi(e): e \in E\}, \quad \phi_{\mathrm{sup}}=\sup \{\phi(e): e \in E\}
$$

If $\phi$ is $\mathcal{H}$-invariant and $\mathcal{H}$ acts quasi-transitively, then

$$
0<\phi_{\text {inf }} \leq \phi_{\text {sup }}<\infty
$$

A walk on $G$ is called $n$-step if it traverses exactly $n$ edges (possibly with reversals and repeats). An $n$-step self-avoiding walk (SAW) on $G$ is an ordered sequence $\pi=$ $\left(\pi_{0}, \pi_{1}, \ldots, \pi_{n}\right)$ of distinct vertices of $G$ such that $e_{i}=\left\langle\pi_{i-1}, \pi_{i}\right\rangle \in E$ for $1 \leq i \leq n$. The weight of $\pi$ is the product

$$
w(\pi):=\prod_{i=1}^{n} \phi\left(\left\langle\pi_{i-1}, \pi_{i}\right\rangle\right) .
$$

Note that the weight function $w=w_{\phi}$ acts symmetrically in that the weight of an edge or SAW is the same irrespective of the direction in which it is traversed. Let $\Sigma_{n}(v)$ be the set of $n$-step SAWs starting at $v \in V$, and set $\Sigma_{n}=\Sigma_{n}(\mathbf{1})$. For a set $\Pi$ of SAWs, we write

$$
w(\Pi)=\sum_{\pi \in \Pi} w(\pi)
$$

for the total weight of members of $\Pi$. 
The following is proved as for the unweighted case in [13] (see also [14,20,23]), and the proof is omitted. For $\mathcal{H} \leq \operatorname{Aut}(G)$, we let $\Phi_{E}(\mathcal{H})$ be the space of functions $\phi: E \rightarrow(0, \infty)$ that are $\mathcal{H}$-invariant.

Theorem 2.1 Let $G=(V, E) \in \mathcal{G}$, and let $\mathcal{H} \leq \operatorname{Aut}(G)$ act quasi-transitively on $G$. Let $\phi \in \Phi_{E}(\mathcal{H})$. There exists $\mu=\mu(G, \phi) \in(0, \infty)$, called the connective constant, such that

$$
\lim _{n \rightarrow \infty} w\left(\Sigma_{n}\right)^{1 / n}=\mu
$$

Let $G=(V, E) \in \mathcal{G}$. It was shown in [9,12] (see also the review [11]) how to define 'bridge' SAWs on certain general families of graphs, and how to adapt the proof of Hammersley and Welsh [15] to show equality of the connective constant and the bridge constant. Key to this approach is the following notion of a graph height function. See [9,22] for accounts of unimodularity.

Definition 2.2 A graph height function on $G=(V, E) \in \mathcal{G}$ is a pair $(h, \mathcal{H})$ such that:

(a) $h: V \rightarrow \mathbb{Z}$, and $h(\mathbf{1})=0$,

(b) $\mathcal{H} \leq \operatorname{Aut}(G)$ acts quasi-transitively on $G$, and $h$ is $\mathcal{H}$-difference-invariant in that

$$
h(\alpha v)-h(\alpha u)=h(v)-h(u), \quad \alpha \in \mathcal{H}, u, v \in V,
$$

(c) for $v \in V$, there exist neighbours $u$, w such that $h(u)<h(v)<h(w)$.

A graph height function $(h, \mathcal{H})$ is called unimodular if the action of $\mathcal{H}$ on $V$ is unimodular.

Associated with a graph height function $(h, \mathcal{H})$ are two integers $d, r$ which we define next. Let

$$
d=d(h)=\max \{|h(u)-h(v)|: u, v \in V, u \sim v\}
$$

where $u \sim v$ means that $u$ and $v$ are neighbours.

If $\mathcal{H}$ acts transitively, we set $r=0$. Assume $\mathcal{H}$ does not act transitively, and let $r=r(h, \mathcal{H})$ be the infimum of all $r$ such that the following holds. Let $o_{1}, o_{2}, \ldots, o_{M}$ be representatives of the orbits of $\mathcal{H}$. For $i \neq j$, there exists $v_{j} \in \mathcal{H} o_{j}$ such that $h\left(o_{i}\right)<h\left(v_{j}\right)$, and a SAW $v_{i, j}:=v\left(o_{i}, v_{j}\right)$ from $o_{i}$ to $v_{j}$, with length $r$ or less, all of whose vertices $x$, other than its endvertices, satisfy $h\left(o_{i}\right)<h(x)<h\left(v_{j}\right)$. We fix such SAWs $v_{i, j}$, and we set $v_{i, i}=\left\{o_{i}\right\}$. These SAWs will be used in Sects. 5 and 6. Meanwhile, set

$$
\phi_{v}=\min \left\{\phi(e): e \in v_{i, j} \text { for some } i, j\right\}
$$

and

$$
\theta_{i, j}=w\left(v_{i, j}\right), \quad \theta_{\min }=\min _{i, j} \theta_{i, j}, \quad \theta_{\max }=\max _{i, j} \theta_{i, j},
$$


where $\theta_{i, i}:=1$. If $\mathcal{H}$ acts transitively, we set $\phi_{v}=\theta_{\min }=\theta_{\max }=1$. Some properties of $r$ and $d$ have been established in [12, Prop. 3.2].

We turn to so-called half-space walks and bridges. Let $G=(V, E) \in \mathcal{G}$ have a graph height function $(h, \mathcal{H})$, and let $\phi \in \Phi_{E}(\mathcal{H})$. Let $v \in V$ and $\pi=$ $\left(\pi_{0}, \pi_{1}, \ldots, \pi_{n}\right) \in \Sigma_{n}(v)$. We call $\pi$ a half-space $S A W$ if

$$
h\left(\pi_{0}\right)<h\left(\pi_{i}\right), \quad 1 \leq i \leq n,
$$

and we write $H_{n}(v)$ for the set of half-space walks $\pi$ with initial vertex $v$. We call $\pi$ a bridge if

$$
h\left(\pi_{0}\right)<h\left(\pi_{i}\right) \leq h\left(\pi_{n}\right), \quad 1 \leq i \leq n,
$$

and a reversed bridge if (2.6) is replaced by

$$
h\left(\pi_{n}\right) \leq h\left(\pi_{i}\right)<h\left(\pi_{0}\right), \quad 1 \leq i \leq n .
$$

The span of a SAW $\pi$ is defined as

$$
\operatorname{span}(\pi)=\max _{0 \leq i \leq n} h\left(\pi_{i}\right)-\min _{0 \leq i \leq n} h\left(\pi_{i}\right)
$$

Let $\beta_{n}(v)$ be the set of $n$-step bridges $\pi$ from $v$, and let

$$
w\left(\beta_{n}\right):=\min \left\{w\left(\beta_{n}(v)\right): v \in V\right\} .
$$

It is easily seen (as in [15]) that

$$
w\left(\beta_{m+n}\right) \geq w\left(\beta_{m}\right) w\left(\beta_{n}\right)
$$

from which we deduce the existence of the bridge constant

$$
\beta=\beta(G, h, \mathcal{H})=\lim _{n \rightarrow \infty} w\left(\beta_{n}\right)^{1 / n}
$$

satisfying

$$
w\left(\beta_{n}\right) \leq \beta^{n}, \quad n \geq 0 .
$$

Remark 2.3 The bridge constant $\beta$ depends on the choice of graph height function $(h, \mathcal{H})$ and weight function $\phi \in \Phi_{E}(\mathcal{H})$. We shall see in Theorem 2.5 that its value is constant (and equal to $\mu$ ) across the set of unimodular graph height functions $(h, \mathcal{H})$ for which $\phi$ is $\mathcal{H}$-invariant.

Proposition 2.4 Let $G=(V, E) \in \mathcal{G}$ have a graph height function $(h, \mathcal{H})$, and suppose $\phi \in \Phi_{E}(\mathcal{H})$. Then

$$
w\left(\beta_{n}(v)\right)^{1 / n} \rightarrow \beta, \quad v \in V
$$


and furthermore

$$
w\left(\beta_{n}(v)\right) \leq \beta^{n}\left(\beta / \phi_{\nu}\right)^{r}, \quad n \geq 1, v \in V
$$

where $r=r(h, \mathcal{H})$ and $\phi_{\nu}$ are given after $(2.3)$.

Theorem 2.5 (Weighted bridge theorem) Let $G=(V, E) \in \mathcal{G}$ possess a unimodular graph height function $(h, \mathcal{H})$, and let $\phi \in \Phi_{E}(\mathcal{H})$. Then $\beta=\mu$.

The proof of Theorem 2.5 is summarized in Sect. 8. It is essentially that of [12, Thm 1]. The supremum metric of the next theorem will appear in a more general form in (4.1).

Remark 2.6 (Added on final revision) Let $G \in \mathcal{G}$ have graph height function $(h, \mathcal{H})$, and consider unweighted SAWs on $G$. Since the current paper was written, Lindorfer [21] has found an expression for the connective constant $\mu=\mu(G)$ without assuming unimodularity, namely $\mu=\max \left\{\beta^{+}, \beta^{-}\right\}$where $\beta^{ \pm}$is the bridge constant associated with the height function $( \pm h, \mathcal{H})$. We have by Grimmett and $\mathrm{Li}$ [12] that $\beta^{+}=\beta^{-}$ if $(h, \mathcal{H})$ is unimodular. Furthermore, unimodularity is necessary for $\beta^{+}=\beta^{-}$, as exemplified by the natural graph height function on Trofimov's grandparent graph [24]. Corresponding statements are valid in the weighted case also.

Theorem 2.7 Let $G=(V, E) \in \mathcal{G}$ have a graph height function $(h, \mathcal{H})$. We endow $\Phi_{E}(\mathcal{H})$ with the supremum metric

$$
d_{\text {sup }}(\phi, \psi)=\sup \{|\phi(e)-\psi(e)|: e \in E\} .
$$

The constants $\mu$ and $\beta$ are continuous functions on $\Phi_{E}(\mathcal{H})$. More precisely,

$$
\left(1-\frac{d_{\text {sup }}(\psi, \phi)}{\phi_{\text {inf }}}\right) \leq \frac{\mu(G, \psi)}{\mu(G, \phi)}, \frac{\beta(G, \psi)}{\beta(G, \phi)} \leq\left(1+\frac{d_{\text {sup }}(\psi, \phi)}{\phi_{\text {inf }}}\right), \quad \phi, \psi \in \Phi_{E}(\mathcal{H})
$$

Proof of Proposition 2.4 Assume $G$ has graph height function $(h, \mathcal{H})$. If $G$ is transitive, the claim is trivial by (2.9) and (2.10), so we assume $G$ is quasi-transitive but not transitive.

Choose $x \in V$ such that $w\left(\beta_{n+r}(x)\right)=w\left(\beta_{n+r}\right)$. Let $v \in V$, and let $v$ have type $o_{j}$ and $x$ type $o_{i}$. Let $v\left(o_{i}, v_{j}\right)$ be given as above (2.4). The length $l\left(o_{i}, v_{j}\right)$ of $v\left(o_{i}, v_{j}\right)$ satisfies $l\left(o_{i}, v_{j}\right) \leq r$. Find $\eta \in \mathcal{H}$ such that $\eta\left(v_{j}\right)=v$, and let $v(x, v)=\eta\left(v\left(o_{i}, v_{j}\right)\right)$. Let $l=l\left(o_{i}, v_{j}\right)$ if $i \neq j$, and $l=0$ otherwise. Then,

$$
\phi_{v}^{r} w\left(\beta_{n}(v)\right) \leq \phi_{v}^{r-l} w\left(\beta_{n+l}(x)\right) \leq w\left(\beta_{n+r}(x)\right)=w\left(\beta_{n+r}\right)
$$

and (2.12) follows by (2.10). Limit (2.11) follows by (2.7) and (2.9). 
Proof of Theorem 2.7 We shall show continuity at the point $\phi \in \Phi_{E}(\mathcal{H})$. For $\pi \in \Sigma_{n}$,

$$
\left(1-\frac{d_{\mathrm{sup}}(\psi, \phi)}{\phi_{\mathrm{inf}}}\right)^{n} \leq \frac{w_{\psi}(\pi)}{w_{\phi}(\pi)}=\prod_{e \in \pi} \frac{\psi(e)}{\phi(e)} \leq\left(1+\frac{d_{\mathrm{sup}}(\psi, \phi)}{\phi_{\mathrm{inf}}}\right)^{n},
$$

from which (2.14) follows.

\section{Weight functions with unbounded support}

We consider weighted SAWs on groups in this section. If the weight function has bounded support, the relevant graph is locally finite, and the methods and conclusions of the last section apply. New methods are needed if the support is unbounded, and it turns out to be useful to consider a different measure of the length of a walk. We shall consider groups that support graph height functions.

A group $\Gamma$ is called indicable if there exists a surjective homomorphism $F: \Gamma \rightarrow \mathbb{Z}$. It is called virtually indicable if there exists $\mathcal{H} \unlhd \Gamma$ with $[\Gamma: \mathcal{H}]<\infty$ such that $\mathcal{H}$ is indicable. If this holds, we call $\Gamma$ virtually $\mathcal{H}$-indicable (or, sometimes, virtually $(\mathcal{H}, F)$-indicable). (See, for example, $[17,18]$ for information on virtual indicability.)

We remind the reader of the definition of a strong graph height function, as derived from [10, Defn 3.4].

Definition 3.1 A (not necessarily locally finite) Cayley graph $G=(\Gamma, F)$ of a finitely generated group $\Gamma$ is said to have a strong graph height function $(h, \mathcal{H})$ if the following two conditions hold.

(a) $\mathcal{H} \unlhd \Gamma$ acts on $\Gamma$ by left multiplication, and $[\Gamma: \mathcal{H}]<\infty$.

(b) $(h, \mathcal{H})$ is a graph height function on $G$.

The group properties of: (i) virtual indicability and (ii) the possession of a strong graph height function, are equivalent in the sense of the next theorem.

Theorem 3.2 Let $\Gamma$ be a finitely generated group.

(a) If $\Gamma$ is virtually $(\mathcal{H}, F)$-indicable, any (not necessarily locally finite) Cayley graph $G=(\Gamma, F)$ of $\Gamma$ possesses a strong graph height function of the form $(h, \mathcal{H})$.

(b) If some Cayley graph $G$ of $\Gamma$ possesses a strong graph height function, denoted $(h, \mathcal{H})$, then $\Gamma$ is virtually $\mathcal{H}$-indicable.

In the context of this theorem, $\mathcal{H}$ acts freely on the Cayley graph $G$ and is therefore unimodular. Hence, the functions $(h, \mathcal{H})$ are unimodular graph height functions on $G$. The proofs of this and other results in this section may be found in Sect. 5 .

Here is some notation. Let $\Gamma$ be finitely generated and virtually $(\mathcal{H}, F)$-indicable. It is shown in [9, Sect. 7] that, for a given locally finite Cayley graph $G$, there exists a unique harmonic extension $\psi$ of $F$ and that $\psi$ takes rational values. It is then shown how to construct a harmonic, strong graph height function for $G$ of the form $(h, \mathcal{H})$. The latter construction is not unique. For given $(\Gamma, \mathcal{H}, F, G)$, we write $h=h_{F}$ for a given such function. 
By Theorems 2.5 and 3.2, the weighted bridge constant equals the weighted connective constant for any locally finite, weighted Cayley graph of a finitely generated, virtually indicable group. Here is an example of a class of such groups.

Example 3.3 (Elementary amenability) Let EFG denote the class of infinite, finitely generated, elementary amenable groups. It is standard that any $\Gamma \in \mathrm{EFG}$ is virtually indicable. See, for example, [9, Proof of Thm 4.1].

We turn to weight functions and SAWs. Let $\Gamma$ be virtually $\mathcal{H}$-indicable, and let $\phi: \Gamma \rightarrow[0, \infty)$ satisfy $\phi(\mathbf{1})=0$ and be symmetric in that

$$
\phi\left(\gamma^{-1}\right)=\phi(\gamma), \quad \gamma \in \Gamma
$$

The support of $\phi$ is the set $\operatorname{supp}(\phi):=\{\gamma: \phi(\gamma)>0\}$. We call $\phi$ summable if

$$
w(\Gamma):=\sum_{\gamma \in \Gamma} \phi(\gamma)
$$

satisfies $w(\Gamma)<\infty$. We write

$$
\phi_{\text {sup }}=\sup \{\phi(\gamma): \gamma \in \Gamma\}
$$

When $\phi$ is summable, we have that

$$
\phi_{\text {sup }}<\infty, \quad|\{\gamma \in \Gamma: \phi(\gamma) \geq C\}|<\infty \text { for } C>0
$$

We misuse notation by setting $\phi(\alpha, \gamma)=\phi\left(\alpha^{-1} \gamma\right)$ for $\alpha, \gamma \in \Gamma$.

We consider SAWs on the complete graph $K=(\Gamma, E)$ with vertex set $\Gamma$ and weights $w=w_{\phi}$ as in (2.2). We say that $\phi$ spans $\Gamma$ if, for $\eta, \gamma \in \Gamma$, there exists a SAW on $K$ from $\eta$ to $\gamma$ with strictly positive weight. This is equivalent to requiring that $\operatorname{supp}(\phi)$ generates $\Gamma$. Let $\Phi$ be the set of functions $\phi: \Gamma \rightarrow[0, \infty)$ that are symmetric, summable, and which span $\Gamma$, and let $\Phi_{\text {bnd }}$ be the subset of $\Phi$ containing functions $\phi$ with bounded support. Functions in $\Phi$ are called weight functions.

Let $\phi \in \Phi$. Since we shall be interested only in SAWs $\pi$ with strictly positive weights $w(\pi)$, we shall have use for the subgraph

$$
K_{\phi}=\left(\Gamma, E_{\phi}\right) \text { where } E_{\phi}=\left\{\langle u, v\rangle: u^{-1} v \in \operatorname{supp}(\phi)\right\}
$$

Proposition 3.4 Let $\Gamma$ be finitely generated and virtually $\mathcal{H}$-indicable, and let $\phi \in \Phi$. The graph $K_{\phi}$ of (3.3) possesses a strong graph height function of the form $(h, \mathcal{H})$.

Proof This is a corollary of Theorem 3.2(a), since $\operatorname{supp}(\phi)$ is a generator set of $\Gamma$.

We turn to the notion of the length of a walk. Let $\Lambda$ be the space of all functions $\ell: \Gamma \rightarrow(0, \infty)$ satisfying 
(a) $\ell$ is symmetric in that $\ell(\gamma)=\ell\left(\gamma^{-1}\right)$ for $\gamma \in \Gamma$,

(b) $\ell$ satisfies

$$
\ell_{\text {inf }}>0 \text { where } \ell_{\text {inf }}:=\inf \{\ell(\gamma): \gamma \in \Gamma\}
$$

We extend the domain of $\ell \in \Lambda$ to the edge set $E$ by defining the $\ell$-length of $e \in E$ to be

$$
\ell(e)=\ell\left(u^{-1} v\right), \quad e=\langle u, v\rangle \in E .
$$

The $\ell$-length of a SAW $\pi=\left(\pi_{0}, \pi_{1}, \ldots, \pi_{n}\right)$ is thus

$$
\ell(\pi)=\sum_{i=1}^{n} \ell\left(e_{i}\right), \quad e_{i}=\left\langle\pi_{i-1}, \pi_{i}\right\rangle .
$$

Evidently,

$$
n \leq \ell(\pi) / \ell_{\text {inf }}
$$

Example 3.5 Let $\phi \in \Phi$ and suppose $\ell \equiv 1$. Then $\ell$ restricted to $K_{\phi}$ is the usual graph distance.

Example 3.6 Let $\phi \in \Phi$, and suppose $\ell(\gamma)=1 / \phi(\gamma)$ for $\gamma \in \operatorname{supp}(\phi)$. The values taken by $\ell$ off $\operatorname{supp}(\phi)$ are immaterial to the study of weighted SAWs $\pi$, since $w_{\phi}(\pi)=$ 0 if $\pi$ traverses any edge not in $\operatorname{supp}(\phi)$.

Let $\Gamma$ be virtually $(\mathcal{H}, F)$-indicable, and let $\left(h_{F}, \mathcal{H}\right)$ be the strong graph height function constructed after Theorem 3.2. A SAW $\pi$ on $K$ is a bridge (with respect to $h_{F}$ ) if (2.6) holds. Let $\phi \in \Phi$ and $\ell \in \Lambda$. For $v \in V$, let $\Sigma(v)$ (respectively, $B(v)$ ) be the set of SAWs (respectively, bridges) on $G$ starting at $v$, and abbreviate $\Sigma(\mathbf{1})=\Sigma$. For $c>0$, let

$$
\begin{aligned}
& \sigma_{m, c}^{\ell}(v)=\{\pi \in \Sigma(v): m \leq \ell(\pi)<m+c\}, \\
& \beta_{m, c}^{\ell}(v)=\{\pi \in B(v): m \leq \ell(\pi)<m+c\} .
\end{aligned}
$$

We shall abbreviate $\sigma_{m, c}^{\ell}(v)$ (respectively, $\beta_{m, c}^{\ell}(v)$ ) to $\sigma_{m, c}(v)$ (respectively, $\beta_{m, c}(v)$ ) when confusion is unlikely to arise. Since $K$ is transitive and $\ell$ is $\Gamma$-invariant, $\sigma_{m, c}(v)$ does not depend on the choice of $v$, and we write $\sigma_{m, c}$ for its common value. In contrast, $\beta_{m, c}(v)$ may depend on $v$ since the height function $h$ is generally only $\mathcal{H}$-differenceinvariant. Although we shall take limits as $m \rightarrow \infty$ through the integers, it will be useful sometimes to allow $m$ to be non-integral in (3.6).

Theorem 3.7 Let $\Gamma$ be finitely generated and virtually $(\mathcal{H}, F)$-indicable with strong graph height function $h_{F}$, and let $\phi \in \Phi$ and $\ell \in \Lambda$. 
(a) For all sufficiently large $c$, the limits

$$
\mu_{\phi, \ell}=\limsup _{m \rightarrow \infty} w_{\phi}\left(\sigma_{m, c}\right)^{1 / m}, \quad \beta_{\phi, \ell}=\lim _{m \rightarrow \infty} w_{\phi}\left(\beta_{m, c}(v)\right)^{1 / m}, \quad v \in \Gamma,
$$

exist and are independent of the choice of $c$ (and of the choice of $v$, in the latter case). They are called the (weighted) connective constant and (weighted) bridge constant, respectively.

(b) If $\ell_{\text {sup }}:=\sup \{\ell(\gamma): \gamma \in \Gamma, \gamma \in \operatorname{supp}(\phi)\}$ satisfies $\ell_{\text {sup }}<\infty$, then lim sup may be replaced by lim in (3.7).

(c) We have that

$$
0<\beta_{\phi, \ell} \leq \mu_{\phi, \ell} \leq \max \left\{1, w(\Gamma)^{1 / \ell_{\text {inf }}}\right\}
$$

Example 3.8 (A non-summable weight function) It is possible to have $w(\Gamma)=\infty$ and $\mu_{\phi, \ell}<\infty$. Let $\Gamma=\mathbb{Z}$, and let $K=(\mathbb{Z}, E)$ be the complete graph on $\Gamma$. Take $\ell$ and $\phi$ by

$$
\ell(n)=|n|, \quad \phi(n)=\frac{1}{|n|}, \quad n \in \mathbb{Z},
$$

whence $w(\Gamma)=\infty$. The edge $\langle m, n\rangle \in E$ has length $\ell(\langle m, n\rangle)=|m-n|$. Let $G_{0}=\left(\mathbb{Z}, E_{0}\right)$, where $E_{0}$ is the set of unordered pairs $m, n$ with $|m-n|=1$. Let $\pi=\left(n_{0}=0, n_{1}, n_{2}, \ldots, n_{k}\right)$ be a SAW on $K$ with $\ell$-length $\ell(\pi)=m$. For $1 \leq i \leq k$, let $\nu_{i}$ be the shortest path on $G_{0}$ from $n_{i-1}$ to $n_{i}$, and let $\tilde{\pi}$ be the $m$-step path on $G_{0}$ obtained by following the paths $v_{1}, v_{2}, \ldots, v_{k}$ in sequence. There are $2^{m}$ $m$-step walks on $G_{0}$ from 0 , and each such walk $\tilde{\pi}$ arises (as above) from at most $2^{m}$ distinct SAWs $\pi$ on $K$. Since each SAW $\pi$ on $K$ has weight satisfying $w(\pi) \leq 1$, we have $w\left(\sigma_{m, 1}\right) \leq 4^{m}$, so that $\mu_{\phi, \ell} \leq 4$.

We have no proof in general of the full convergence $w_{\phi}\left(\sigma_{m, c}\right)^{1 / m} \rightarrow \mu_{\phi, \ell}$, but we shall see in Theorem 3.10 that this holds subject to an additional condition.

Theorem 3.7(a) assumes a lower bound on the length $c$ of the intervals in (3.6). That some lower bound is necessary is seen by considering Example 3.6 with a weight function $\phi$ every nonzero value of which has the form $2^{-s}$ for some integer $s \geq 1$. When this holds, every $\ell$-length is a multiple of 2 , implying that $\sigma_{m, c}, \beta_{m, c}=\varnothing$ if $m$ is odd and $c<1$. The required lower bounds on $c$ are discussed after the statement of Proposition 5.4; see (5.3).

We introduce next a condition on the triple $\left(\phi, \ell, h_{F}\right)$. Let $\Gamma$ be virtually $(\mathcal{H}, F)$ indicable with strong graph height function $\left(h_{F}, \mathcal{H}\right)$, and let $\phi \in \Phi$. For $\epsilon \in[1,2)$ and $C>0$, let $\Lambda_{\epsilon}=\Lambda_{\epsilon}(C, \phi)$ be the subset of $\Lambda$ containing functions $\ell$ satisfying the following Hölder condition for the height function:

$$
\left|h_{F}(u)-h_{F}(v)\right| \leq C\left[\ell\left(u^{-1} v\right)\right]^{\epsilon}, \quad u, v \in \Gamma, \phi\left(u^{-1} v\right)>0 .
$$


Note that the usual graph distance of Example 3.6 satisfies (3.9) (with $\epsilon=1$ and suitable $C$ ) if $\phi$ has bounded support; the converse is false, as illustrated in Example 3.13.

Example 3.9 Let $\Gamma$ be finitely generated with finite generating set $S$ and graph height function $(h, \mathcal{H})$, and let $G_{S}$ be the corresponding Cayley graph of $\Gamma$. For $u \in \Gamma$, let $\ell(u)$ be the number of edges in the shortest path of $G_{S}$ from 1 to $u$. Then $\ell$ satisfies (3.9) with $\epsilon=1$ and suitable $C$. Readers may prefer to fix their ideas on such $\ell$. Property (3.9) will feature briefly in the proofs of the ensuing theorems.

Here is our first main theorem, proved in Sect. 6. Once again, we require $c \geq A$ where $A$ will be given in (5.3).

Theorem 3.10 Let $\Gamma$ be finitely generated and virtually $(\mathcal{H}, F)$-indicable with strong graph height function $\left(h_{F}, \mathcal{H}\right)$, and let $\phi \in \Phi$ and $\ell \in \Lambda_{\epsilon}(C, \phi)$ for some $\epsilon \in[1,2)$ and $C>0$.

(a) We have that $\mu_{\phi, \ell}=\beta_{\phi, \ell}$, and furthermore, for all sufficiently large $c$,

$$
\mu_{\phi, \ell}=\lim _{m \rightarrow \infty} w_{\phi}\left(\sigma_{m, c}\right)^{1 / m}
$$

(b) (Monotonicity of $\mu$ ) If $v \in \Phi$ and $v \leq \phi$, then $\ell \in \Lambda_{\epsilon}(C$, $v)$ and $\mu_{v, \ell} \leq \mu_{\phi, \ell}$.

A sufficient condition for (3.9) is presented in Lemma 5.2.

Remark 3.11 (Condition (3.9)) Some condition of type (3.9) is necessary for the proof that $\mu_{\phi, \ell}=\beta_{\phi, \ell}$ (presented in Sect. 6) for the following reason. A key estimate in the proof of [15] concerning the bridge constant is the classical Hardy-Ramanujan [16] estimate of $\exp (\pi \sqrt{n / 3})$ for the number of ordered partitions of an integer $n$. It is important for the proof that this number is $e^{\mathrm{o}(n)}$. Inequality (3.9) implies that the aggregate height difference along a SAW $\pi$ has order no greater than $n:=\left\lfloor(\ell(\pi))^{\epsilon}\right\rfloor$ with $\epsilon<2$, which has $\exp (\mathrm{o}(\ell(\pi)))$ ordered partitions. (See Proposition 5.6.)

Remark 3.12 (Working with graph distance) Let us work with the graph distance of Example 3.5 (so that the length of a walk is the number of its edges), with $\Sigma_{n}$ and $\beta_{n}$ given as usual. Then $w\left(\Sigma_{n}\right)$ is sub-multiplicative, whence the limit $\mu=\lim _{n \rightarrow \infty} w\left(\Sigma_{n}\right)^{1 / n}$ exists. By Theorem 3.7(c), $\mu<\infty$ if $w(\Gamma)<\infty$. It is not hard to see the converse, as follows. Suppose $w(\Gamma)<\infty$. Let $\pi=\left(\pi_{0}=\mathbf{1}, \pi_{1}, \ldots, \pi_{n}\right)$ be a SAW with $w(\pi)>0$, and write $\Gamma^{\prime}=\Gamma \backslash\left\{\pi_{j}^{-1}: 0 \leq j \leq n\right\}$. Then $\Sigma_{n+1}$ contains the walks $\left\{\gamma+\pi: \gamma \in \Gamma^{\prime}\right\}$, where $\gamma+\pi:=\left(\mathbf{1}, \gamma, \gamma \pi_{1}, \ldots, \gamma \pi_{n}\right)$. Therefore, $w\left(\Sigma_{n+1}\right) \geq w\left(\Gamma^{\prime}\right) w(\pi)=\infty$ for $n \geq 1$.

We have no general proof of the equality of $\mu$ and the bridge limit $\beta$ when $\operatorname{supp}(\phi)$ is unbounded, although this holds subject to (3.9) with $\ell$ taken as the graph distance of Example 3.5.

Example 3.13 (Still working with graph distance) Let $\phi \in \Phi$ and take $\ell$ to be graph distance on $K_{\phi}$, as in Example 3.5. By Theorem 3.7(b), the limit $\mu_{\phi, \ell}=$ 
$\lim _{m \rightarrow \infty} w\left(\sigma_{m, c}\right)^{1 / m}$ exists, and it is easy to see that one may take any $c>0$. Here is an example in which $K_{\phi}$ is not locally finite. Let $\Gamma=\mathbb{Z}^{2}$, and let

$$
\phi(\langle p, q\rangle)= \begin{cases}\left(|p|^{2}+|q|^{2}\right)^{-1} & \text { if either } p=0, \text { or }(p, q)=( \pm 1,0) \\ 0 & \text { otherwise }\end{cases}
$$

Then $K_{\phi}$ is the Cayley graph of $\Gamma$ in which $(m, n)$ and $\left(m^{\prime}, n^{\prime}\right)$ are joined by an edge if and only if either $m=m^{\prime}$ or $\left(m^{\prime}, n^{\prime}\right)=(m \pm 1, n)$. Define the height function by $h(m, n)=m$, and note that (3.9) holds for suitable $C$.

Remark 3.14 Let $\Gamma$ be finitely generated and virtually $(\mathcal{H}, F)$-indicable, and let $\phi \in \Phi$ and $\ell \in \Lambda_{\epsilon}(C, \phi)$ for some $\epsilon \in[1,2)$ and $C>0$. Since $\mu_{\phi, \ell}$ is independent of the choice of triple $\left(h_{F}, \mathcal{H}, F\right)$, so is $\beta_{\phi, \ell}$.

\section{Continuity of the connective constant}

A continuity theorem for connective constants is proved in this section. We work on the space $\Phi \times \Lambda$ with distance function

$$
D_{\text {sup }}\left(\left(\phi, \ell_{1}\right),\left(\psi, \ell_{2}\right)\right)=\sup _{\gamma}\left|\frac{\phi(\gamma)-\psi(\gamma)}{\phi(\gamma)+\psi(\gamma)}\right|+\sup _{\gamma}\left|\frac{\ell_{1}(\gamma)-\ell_{2}(\gamma)}{\ell_{1}(\gamma)+\ell_{2}(\gamma)}\right|,
$$

where the suprema are over the set $\operatorname{supp}(\phi) \cup \operatorname{supp}(\psi)$. This may be compared with (2.13).

The following continuity theorem is our second main theorem, and it is proved in Sect. 7. For $C, W>0$ and $\epsilon \in[1,2)$, we write $\Phi \circ \Lambda_{\epsilon}(C, W)$ for the space of all pairs $(\phi, \ell)$ satisfying $\phi \in \Phi, \ell \in \Lambda_{\epsilon}(C, \phi)$, and $\mu_{\phi, \ell} \leq W$.

Theorem 4.1 (Continuity theorem) Let $\Gamma$ be finitely generated and virtually $(\mathcal{H}, F)$ indicable. Let $C, W>0$ and $\epsilon \in[1,2)$. The connective constant $\mu$ is a continuous function on the space $\Phi \circ \Lambda_{\epsilon}(C, W)$ endowed with the distance function $D_{\text {sup }}$ of (4.1).

Section 7 contains also a proposition concerning the effect on a connective constant $\mu_{\phi, \ell}$ of truncating the weight function $\phi$; see Proposition 7.4.

\section{Proofs of Theorems 3.2 and 3.7}

We prove next the aforesaid theorems together with some results in preparation for the proof in Sect. 6 of Theorem 3.10. We suppress explicit reference to $\phi$ and $\ell$ except where necessary to avoid ambiguity.

Lemma 5.1 Let $\Gamma$ be finitely generated and let $G$ be a (not necessarily locally finite) Cayley graph of $\Gamma$ with generator set $S=\left(s_{i}: i \in I\right)$. There exists a locally finite Cayley graph $G^{\prime}$ of $\Gamma$ with (finite) generator set $S^{\prime} \subseteq S$. 
Proof Let $S_{0}$ be a finite generator set of $\Gamma$. Each $s \in S_{0}$ can be expressed as a finite product of the form $s=\prod\left\{\psi: \psi \in T_{s}\right\}$ with $T_{s} \subseteq S$. We set $S^{\prime}=\bigcup_{s \in S_{0}} T_{s}$.

Proof of Theorem 3.2 (a) Let $G$ be a Cayley graph of $\Gamma$ with generator set $S$. By Lemma 5.1, we can construct a locally finite Cayley graph $G^{\prime}$ of $\Gamma$ with respect to a finite set $S^{\prime} \subseteq S$ of generators. Since $\mathcal{H}$ acts freely and is therefore unimodular, we may apply [9, Thm 3.4] to deduce that $G^{\prime}$ has a strong graph height function of the form $(h, \mathcal{H})$. Since $G^{\prime}$ is a subgraph of $G$ with the same vertex set, and $h$ is $\mathcal{H}$-difference-invariant on $G^{\prime}$, it is $\mathcal{H}$-difference-invariant on $G$ also.

It remains to check that each vertex $v \in \Gamma$ has neighbours $u, w$ in $G$ with $h(u)<$ $h(v)<h(w)$. This holds since there exist $s_{1}, s_{2} \in S^{\prime} \subseteq S$ such that $h\left(v s_{1}\right)<h(v)<$ $h\left(v s_{2}\right)$.

(b) Let $G$ be a Cayley graph of $\Gamma$ with a strong graph height function $(h, \mathcal{H})$. Then $\mathcal{H} \unlhd \Gamma$ and $[\Gamma: \mathcal{H}]<\infty$. Let $\lambda=\operatorname{gcd}\{|h(u)|: u \in \mathcal{H}, h(u) \neq 0\}$. Then $\Gamma$ is virtually $(\mathcal{H}, F)$-indicable with $F=h / \lambda$.

There follows a sufficient condition for condition (3.9) of the principal Theorem 3.10.

Lemma 5.2 Let $\Gamma$ be finitely generated and $\phi \in \Phi$. Let $G$ be the Cayley graph with generator set $\operatorname{supp}(\phi)$, and let $G^{\prime}$ be as in Lemma 5.1. Write $\delta^{\prime}$ for graph distance on $G^{\prime}$. Let $\left(h_{F}, \mathcal{H}\right)$ be the strong graph height function described after Theorem 3.2. If there exists $C_{1}>0$ and $\epsilon \in[1,2)$ such that

$$
\ell(v) \geq C_{1} n^{1 / \epsilon}, \quad v \in \Gamma, \delta^{\prime}(\mathbf{1}, v)=n \geq 1,
$$

then there exists $C>0$ such that (3.9) holds.

Proof Since $\left(h_{F}, \mathcal{H}\right)$ is a graph height function on the locally finite graph $G^{\prime}$, by (2.3) there exists $d \in(0, \infty)$ such that

$$
\left|h_{F}(u)-h_{F}(v)\right| \leq d n, \quad u, v \in \Gamma, \delta^{\prime}(u, v)=n .
$$

Inequality (3.9) follows by (5.1) with $C=d C_{1}^{-\epsilon}$.

Lemma 5.3 Let $\Gamma$ be finitely generated and virtually $\mathcal{H}$-indicable, where $\mathcal{H} \neq \Gamma$, and let $\phi \in \Phi$. Let $K_{\phi}=\left(\Gamma, E_{\phi}\right)$ be as in (3.3), and let $(h, \mathcal{H})$ be a strong graph height function on $K_{\phi}$. Let $o_{i}$ be a representative of the ith orbit of $\mathcal{H}$. There exists an integer $s=s(h, \mathcal{H}, \phi) \in \mathbb{N}$ such that the following holds. For $i \neq j$, there exists $v_{j} \in \mathcal{H} o_{j}$ such that $h\left(o_{i}\right)<h\left(v_{j}\right)$, and a $S A W v_{i, j}=v\left(o_{i}, v_{j}\right)$ of $G^{\prime}$ from $o_{i}$ to $v_{j}$ with $\ell$-length $s$ or less, each of whose vertices $x$ other than its endvertices satisfies $h\left(o_{i}\right)<h(x)<h\left(v_{j}\right)$.

Proof This extends to $K_{\phi}$ the discussion above (2.4). Let $G^{\prime}$ be a locally finite Cayley graph of $\Gamma$ with respect to a finite generator set $S \subseteq \operatorname{supp}(\phi)$, as in Lemma 5.1. By Grimmett and $\mathrm{Li}$ [12, Prop. 3.2], there exists $0<r<\infty$ such that, for $i \neq j$, there exists $v_{j} \in \mathcal{H} o_{j}$ such that $h\left(o_{i}\right)<h\left(v_{j}\right)$, and a SAW $v_{i, j}=v\left(o_{i}, v_{j}\right)$ on $G^{\prime}$ from 
$o_{i}$ to $v_{j}$ with graph length $r$ or less. Furthermore, each of the vertices $x$ of $v\left(o_{i}, v_{j}\right)$ other than its endvertices satisfies $h\left(o_{i}\right)<h(x)<h\left(v_{j}\right)$.

Since $G^{\prime}$ is a subgraph of $G$, a SAW on $G^{\prime}$ is also a SAW on $G$. Moreover, since $\mathcal{H}$ acts quasi-transitively, and every edge of $G^{\prime}$ has finite $\ell$-length, we can find $s \in(0, \infty)$ such that the claim holds.

Lemma 5.4 Let $\Gamma$ be finitely generated and virtually $(\mathcal{H}, F)$-indicable with strong graph height function $h_{F}$, and let $\phi \in \Phi$ and $\ell \in \Lambda$. There exist $\beta_{\phi, \ell} \in(0, \infty)$ and $A=A(\phi, \mathcal{H}, F)$ such that, for $c \geq A$,

$$
\lim _{m \rightarrow \infty} w\left(\beta_{m, c}(v)\right)^{1 / m}=\beta_{\phi, \ell}, \quad v \in \Gamma .
$$

The constant $A$ is given in forthcoming equations (5.3).

In advance of the proof, we introduce some notation that will be useful later in this work. Let $G^{\prime}=\left(\Gamma, E^{\prime}\right)$ be the Cayley graph generated by the finite $S^{\prime} \subseteq \operatorname{supp}(\phi)$ of Lemma 5.1 applied to $G:=K_{\phi}$. By Theorem 3.2(a) and its proof, there exists $\left(h_{F}, \mathcal{H}\right)$ which is a strong graph height function of both $K_{\phi}$ and $G^{\prime}$.

Since $h_{F}$ is a graph height function on $G^{\prime}$, for $v \in \Gamma$, there exists an edge $e_{v}=$ $\left\langle v, v \alpha_{v}\right\rangle \in E^{\prime}$ such that $h_{F}\left(v \alpha_{v}\right)>h_{F}(v)$ (and also $\phi\left(\alpha_{v}\right)>0$ by definition of $G^{\prime}$ ). We call the edge $e_{v}$ the extension at $v$. Write

$$
\begin{aligned}
& \psi=\inf \left\{\phi\left(\alpha_{v}\right): v \in \Gamma\right\}, \\
& a=\inf \left\{\ell\left(\alpha_{v}\right): v \in \Gamma\right\}, \quad A=\sup \left\{\left\lceil\ell\left(\alpha_{v}\right)\right\rceil: v \in \Gamma\right\},
\end{aligned}
$$

noting that $\psi, a>0$ and $A<\infty$ since $h_{F}$ is $\mathcal{H}$-difference-invariant and $\mathcal{H}$ acts quasi-transitively. Let

$$
w\left(\beta_{m, A}\right)=\inf \left\{w\left(\beta_{m, A}(v)\right): v \in \Gamma\right\} .
$$

Lemma 5.5 For $q \geq c-A \geq 0$, there exists $C_{1}=C_{1}(\psi, q, c, a) \in(0, \infty)$ such that

$$
w\left(\beta_{p, c}(v)\right) \leq C_{1} w\left(\beta_{p+q, A}(v)\right), \quad p \in(0, \infty), v \in \Gamma
$$

Proof Let $\pi \in \beta_{p, c}(v)$. We may extend $\pi$ by adding progressive extensions at the final endpoint. After some number $r$ of such extensions, we achieve a bridge $\pi^{\prime}$ contributing to $\beta_{p+q, A}$. Note that $w\left(\pi^{\prime}\right) \geq \psi^{r} w(\pi)$, and $r \leq\lceil q / a\rceil$. Each such $\pi^{\prime}$ occurs no more than $\lceil c / a\rceil$ times in this construction. The claimed inequality holds with $C_{1}=\lceil c / a\rceil \psi^{-\lceil q / a\rceil}$.

Proof of Lemma 5.4 By concatenation as usual,

$$
w\left(\beta_{m, A}\right) w\left(\beta_{n . A}\right) \leq w\left(\beta_{m+n, 2 A}(v)\right), \quad v \in \Gamma .
$$

By Lemma 5.5, there exists $C=C(\psi, A, a)$ such that

$$
w\left(\beta_{m, A}\right) w\left(\beta_{n, A}\right) \leq C w\left(\beta_{m+n+A, A}(v)\right) .
$$


This holds for all $v$, whence

$$
w\left(\beta_{m, A}\right) w\left(\beta_{n, A}\right) \leq C w\left(\beta_{m+n+A, A}\right) .
$$

We deduce (by subadditivity, as in [8, eqn (8.38)], for example) the existence of the limit

$$
\beta_{\phi, \ell}=\beta_{\phi, \ell, A}:=\lim _{m \rightarrow \infty} w\left(\beta_{m, A}\right)^{1 / m}
$$

In particular, by (5.4),

$$
\liminf _{m \rightarrow \infty} w\left(\beta_{m, A}(v)\right)^{1 / m} \geq \beta_{\phi, \ell}, \quad v \in \Gamma .
$$

By (5.5),

$$
w\left(\beta_{m, A}\right) \leq C^{-1}\left(\beta_{\phi, \ell}\right)^{m+A} .
$$

We show next that (5.2) (with $c=A$ ) is valid for all $v \in \Gamma$. Let $s$ be as in Lemma 5.3, and let $x, v \in \Gamma$ have types $o_{i}, o_{j}$, respectively, where $i \neq j$. Any $b_{v} \in \beta_{m, A}(v)$ may be prolonged 'backwards' by a translate $\alpha v\left(o_{i}, v_{j}\right)$, for suitable $\alpha \in \mathcal{H}$, to obtain a bridge $b_{x}$ from $x$ with weight $w\left(b_{x}\right)=\theta_{i, j} w\left(b_{v}\right)$, where $\theta_{i, j}=w\left(v\left(o_{i}, v_{j}\right)\right)$ as in (2.5). By Lemma 5.5, there exists $C_{1}=C_{1}(\psi, A, a, s)$ such that

$$
\theta_{i, j} w\left(\beta_{m, A}(v)\right) \leq C_{1} w\left(\beta_{m+s, A}(x)\right.
$$

If $i=j$, this holds with $\theta_{i, j}=1$.

We pick $x$ such that $w\left(\beta_{m+s, A}(x)\right)=w\left(\beta_{m+s, A}\right)$. By (5.4) and (5.7), equation (5.2) follows for $v$, with $c=A$, and in addition

$$
w\left(\beta_{m, A}(v)\right) \leq C_{2}\left(\beta_{\phi, \ell}\right)^{m+s+A}, \quad v \in \Gamma,
$$

by (5.7), where $C_{2}=C_{2}\left(\psi, A, a, \theta_{\min }, s\right)$.

Equation (5.2) with $c=A$ follows by (5.6) and (5.8). When $c>A$, it suffices to note that

$$
\beta_{m, A}(v) \subseteq \beta_{m, c}(v) \subseteq \bigcup\left\{\beta_{m+i, A}(v): 0 \leq i \leq\lceil c-A\rceil\right\}
$$

implying that the value of the limit $\beta_{\phi, \ell}$ is independent of $c>A$. By (5.3), $w\left(\beta_{0, A}\right)>$ 0 , and hence $\beta_{\phi, \ell}>0$ by (5.8).

Proof of Theorem 3.7 (a) The second limit in (3.7) is included in Lemma 5.4. That $\mu_{\phi, \ell}$ is independent of the value of $c \geq A$ holds by (5.9) with $\sigma$ substituted for $\beta$.

(b) Let $L=\left\lceil\ell_{\text {sup }}\right\rceil<\infty$. The usual sub-multiplicative argument for SAWs yields 


$$
\begin{aligned}
w\left(\sigma_{m+n, L}\right) & \leq w\left(\sigma_{m, L}\right) w\left(\sigma_{n-L, 2 L}\right) \\
& =w\left(\sigma_{m, L}\right)\left[w\left(\sigma_{n-L, L}\right)+w\left(\sigma_{n, L}\right)\right]
\end{aligned}
$$

By (5.10) with $m=k-L \geq 0$ and $n=L$,

$$
w\left(\sigma_{k, L}\right) \leq w\left(\sigma_{k-L, L}\right)\left[w\left(\sigma_{0, L}\right)+w\left(\sigma_{L, L}\right)\right]
$$

which we substitute in (5.10) with $k=n$ to obtain

$$
w\left(\sigma_{m+n, L}\right) \leq C w\left(\sigma_{m, L}\right) w\left(\sigma_{n-L, L}\right)
$$

with $C=w\left(\sigma_{0, L}\right)+w\left(\sigma_{L, L}\right)$. The claim follows in the usual way with $c=L$. As at (5.7),

$$
w\left(\sigma_{m, L}\right) \geq C^{-1}\left(\mu_{\phi, \ell}\right)^{m+L} .
$$

(c) It is trivial that $\beta_{\phi, \ell} \leq \mu_{\phi, \ell}$, and it was noted at the end of the proof of part (a) that $\beta_{\phi, \ell}>0$. Let $\Sigma_{n}$ be the set of $n$-step SAWs from 1. By (3.5),

$$
w\left(\sigma_{m, c}\right) \leq \sum_{i=1}^{\left\lfloor(m+c) / \ell_{\mathrm{inf}}\right\rfloor} w\left(\Sigma_{i}\right) .
$$

Equation (3.8) follows since $w\left(\Sigma_{i}\right) \leq w(\Gamma)^{i}$.

Proposition 5.6 Assume $\phi \in \Phi$ and $\ell \in \Lambda_{\epsilon}(C, \phi)$ for $\epsilon \in[1,2)$ and $C>0$. For $u, v \in \Gamma$ and any $S A W \pi$ on $K_{\phi}$ from $u$ to $v$,

$$
\left|h_{F}(u)-h_{F}(v)\right| \leq C \ell(\pi)^{\epsilon}
$$

Proof Suppose $\pi$ has vertices $u_{i}:=u \gamma_{1} \gamma_{2} \ldots \gamma_{i}$ for $0 \leq i<n$. Now,

$$
\begin{aligned}
\left|h_{F}(u)-h_{F}(v)\right| & \leq \sum_{i=1}^{n}\left|h_{F}\left(u_{i+1}\right)-h_{F}\left(u_{i}\right)\right| \\
& \leq C \sum_{i=1}^{n} \ell\left(\gamma_{i}\right)^{\epsilon} \text { by }(3.9) \\
& \leq C\left(\sum_{i=1}^{n} \ell\left(\gamma_{i}\right)\right)^{\epsilon}=C \ell(\pi)^{\epsilon},
\end{aligned}
$$

as required. 


\section{Proof of Theorem 3.10}

Let $\phi \in \Phi$ and $\ell \in \Lambda_{\epsilon}(C, \phi)$ with $\epsilon \in[1,2)$ and $C>0$. With the height function $h=h_{F}$ given as after Theorem 3.2, and $A$ as in (5.3), we abbreviate $\beta_{m, A}^{\ell}=\beta_{m}$ and $\sigma_{m, A}^{\ell}=\sigma_{m}$. Clearly, $\beta_{m} \leq \sigma_{m}$, whence $\beta_{\phi, \ell} \leq \mu_{\phi, \ell}$. It suffices, therefore, to show that $\beta_{\phi, \ell} \geq \mu_{\phi, \ell}$. We do this by adapting the proof of [12, Thm 4.3]; it may also be done using the group structure of $\Gamma$ directly, rather than by referring to unimodularity. Let $H_{m}(v)$ be the set of half-space walks from $v$ with $\ell$-lengths in the interval $[m, m+A)$. Theorem 3.10 will follow immediately from Proposition 6.2.

We shall introduce several constants, denoted $D$, which depend on certain parameters as specified. The single character $D$ is used repeatedly for economy of notation; the value of such $D$ can vary between appearances. We recall the constants $\theta_{\min }, \theta_{\max }$ of (2.5), $r$ as after (2.3), $s$ of Lemma 5.3, and $\psi, A, a$ of (5.3).

Proposition 6.1 There exist constants $D=D\left(\psi, A, a, \theta_{\min }, \theta_{\max }, r, s\right)$, which are continuous functions of $\psi, A, a, \theta_{\min }, \theta_{\max }$, such that

$$
w\left(H_{m}(v)\right) \leq D m^{2 \epsilon} e^{D m^{\epsilon / 2}}\left(\beta_{\phi, \ell}\right)^{m+N m^{\epsilon / 2}}, \quad m \geq 1
$$

where

$$
N= \begin{cases}D & \text { if } \beta_{\phi, \ell}>1 \\ 0 & \text { if } \beta_{\phi, \ell} \leq 1\end{cases}
$$

Proposition 6.2 There exist constants $D=D\left(\psi, A, a, \theta_{\min }, \theta_{\max }, r, s\right)$, which are continuous functions of $\psi, A, a, \theta_{\min }, \theta_{\max }$, such that

$$
w\left(\sigma_{m}\right) \leq D m^{4 \epsilon} e^{D m^{\epsilon / 2}}\left(\beta_{\phi, \ell}\right)^{m+N m^{\epsilon / 2}}, \quad m \geq 1,
$$

where $N$ is given in (6.1).

Proof of Proposition 6.1 Let $\pi=\left(\pi_{0}=v, \pi_{1}, \ldots, \pi_{n}\right) \in H_{m}(v)$ have graph length $n$ and $\ell$-length $\ell(\pi)$. Let $n_{0}=0$, and for $j \geq 1$, define $S_{j}=S_{j}(\pi)$ and $n_{j}=n_{j}(\pi)$ recursively as follows:

$$
S_{j}=\max _{n_{j-1} \leq t \leq n}(-1)^{j}\left[h\left(\pi_{n_{j-1}}\right)-h\left(\pi_{t}\right)\right]
$$

and $n_{j}$ is the largest value of $t$ at which the maximum is attained. The recursion is stopped at the smallest integer $k=k(\pi)$ such that $n_{k}=n$, so that $S_{k+1}$ and $n_{k+1}$ are undefined. Note that $S_{1}$ is the span of $\pi$ and, more generally, $S_{j+1}$ is the span of the SAW $\bar{\pi}^{j+1}:=\left(\pi_{n_{j}}, \pi_{n_{j}+1}, \ldots, \pi_{n_{j+1}}\right)$. Moreover, each of the subwalks $\bar{\pi}^{j+1}$ is either a bridge or a reversed bridge. We observe that $S_{1}>S_{2}>\cdots>S_{k}>0$.

For a decreasing sequence of $k \geq 1$ positive integers $a_{1}>a_{2}>\cdots>a_{k}>0$, let $B_{m, c}^{v}\left(a_{1}, a_{2}, \ldots, a_{k}\right)$ be the set of half-space walks $\pi$ from $v \in \Gamma$ with $\ell$-length satisfying $\ell(\pi) \in[m, m+c)$ and such that $k(\pi)=k, S_{1}(\pi)=a_{1}, \ldots, S_{k}(\pi)=$ 
$a_{k}$, and $n_{k}(\pi)=n$ (and hence $S_{k+1}$ is undefined). We abbreviate $B_{m, A}^{v}=B_{m}^{v}$. In particular, $B_{m}^{v}(p)$ is the set of bridges $\pi$ from $v$ with span $p$ and $\ell$-length $\ell(\pi) \in$ $[m, m+A)$.

Recall the weight $\theta_{i, j}$ of the SAW $v_{i, j}=v\left(o_{i}, v_{j}\right)$ as after (2.3). Let $s_{i, j}$ be the $\ell$-length of $v_{i, j}$, and

$$
\delta_{i, j}=h\left(v_{j}\right)-h\left(o_{i}\right), \quad s=\left\lceil\max \left\{s_{i, j}\right\}\right\rceil,
$$

as in Lemma 5.3. We shall perform surgery on $\pi$ to obtain a SAW $\pi^{\prime}$ satisfying

$$
\begin{aligned}
\pi^{\prime} & \in \begin{cases}B_{m+2 s_{i, j}}^{v}\left(a_{1}+a_{2}+a_{3}+2 \delta_{i, j}, a_{4}, \ldots, a_{k}\right) & \text { if } k \geq 3, \\
B_{m+s_{i, j}}^{v}\left(a_{1}+a_{2}+\delta_{i, j}\right) & \text { if } k=2,\end{cases} \\
w\left(\pi^{\prime}\right) & = \begin{cases}\theta_{i, j}^{2} w(\pi) & \text { if } k \geq 3, \\
\theta_{i, j} w(\pi) & \text { if } k=2,\end{cases}
\end{aligned}
$$

for some $i, j$ depending on $\pi$. Note that the subscripts in (6.3) may be non-integral.

The new SAW $\pi^{\prime}$ is constructed in the following way. Suppose first that $k \geq 3$. In the following, we use the fact that $\mathcal{H}$ acts on $\Gamma$ by left multiplication.

1. Let $t=\min \left\{u: h\left(\pi_{u}\right)=a_{1}\right\}$, and let $\pi(1)$ be the sub-SAW from $\pi_{0}=\mathbf{1}$ to the vertex $\pi_{t}$.

2. Let $\sigma:=\left(\pi_{t}, \ldots, \pi_{n_{2}}\right)$ and $\rho=\left(\pi_{n_{2}}, \ldots, \pi_{n}\right)$ be the two sub-SAWs of $\pi$ with the given endvertices. The type of $\pi_{t}$ (respectively, $\pi_{n_{2}}$ ) is denoted $o_{i}$ (respectively, $o_{j}$ ). In particular, $\pi_{t}=\gamma o_{i}$ for some $\gamma \in \mathcal{H}$. We map the SAW $v_{i, j}=v\left(o_{i}, v_{j}\right)$, given after (2.3), under $\gamma$ to obtain a SAW denoted $\nu\left(\pi_{t}, \alpha_{1} \pi_{n_{2}}\right)$, where $\alpha_{1}=\gamma v_{j} \pi_{n_{2}}^{-1}$ is the unique element of $\mathcal{H}$ such that $\alpha_{1} \pi_{n_{2}}=\gamma v_{j}$. Note that $\nu\left(\pi_{t}, \alpha_{1} \pi_{n_{2}}\right)$ is the single point $\left\{\pi_{t}\right\}$ if $i=j$, and $\alpha_{1} \pi_{n_{2}}=\pi_{t}$ in this case.

The union of the three SAWs $\pi(1), v\left(\pi_{t}, \alpha_{1} \pi_{n_{2}}\right)$, and $\sigma^{\prime}:=\alpha_{1} \sigma$ (reversed) is a SAW, denoted $\pi(2)$, from $v$ to $\alpha_{1} \pi_{t}$. Note that $h\left(\alpha_{1} \pi_{t}\right)=a_{1}+a_{2}+\delta_{i, j}$ where $\delta_{i, j}$ is given in (6.2).

3. We next perform a similar construction in order to connect $\alpha_{1} \pi_{t}$ to an image of the first vertex $\pi_{n_{2}}$ of $\rho$. These two vertices have types $o_{i}$ and $o_{j}$, as before, and thus we insert the SAW $v\left(\alpha_{1} \pi_{t}, \alpha_{2} \pi_{n_{2}}\right):=\alpha_{1} \gamma \nu\left(o_{i}, v_{j}\right)$, where $\alpha_{2}=\alpha_{1} \gamma v_{j} \pi_{n_{2}}^{-1}$ is the unique element of $\mathcal{H}$ such that $\alpha_{2} \pi_{n_{2}}=\alpha_{1} \gamma v_{j}$. The union of the three SAWs $\pi(2), v\left(\alpha_{1} \pi_{t}, \alpha_{2} \pi_{n_{2}}\right)$, and $\rho^{\prime}:=\alpha_{2} \rho$ is a SAW denoted $\pi^{\prime}$ from 1 to $\alpha_{2} \pi_{n}$.

The resulting SAW $\pi^{\prime}$ is a half-space SAW from $v$ with $\ell$-length satisfying $\ell\left(\pi^{\prime}\right) \in$ $\left[m+2 s_{i, j}, m+2 s_{i, j}+A\right)$. The maximal bridges and reversed bridges that compose $\pi^{\prime}$ have height changes $a_{1}+a_{2}+a_{3}+2 \delta_{i, j}, a_{4}, \ldots, a_{k}$. Note that $\pi^{\prime}$ is obtained by (i) reordering the steps of $\pi$, (ii) reversing certain steps, and (iii) adding paths with (multiplicative) aggregate $\ell$-weight $\theta_{i, j}^{2}$. In particular, (6.3)-(6.4) hold. If $k=2$, the construction ceases after Step 2.

We consider next the multiplicities associated with the map $\pi \mapsto \pi^{\prime}$. The argument in the proof of [12, Sect. 7] may not be used directly since the graph $K_{\phi}$ is not assumed locally finite. The argument required here is, however, considerably simpler since we are working with Cayley graphs. 


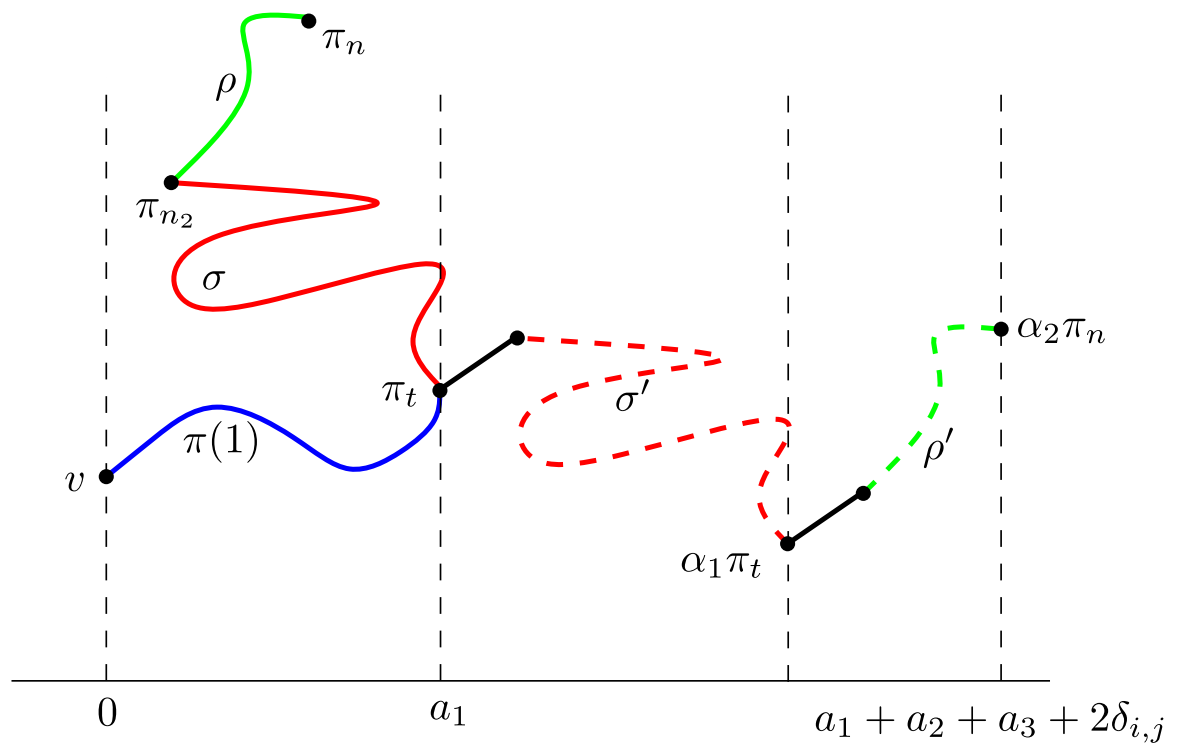

Fig. 1 Two images of $v_{i, j}$ are introduced in order to make the required connections. These extra connections are drawn as black straight-line segments, and each has weight $\theta_{i, j}, \ell$-length $s_{i, j}$, and span $\delta_{i, j}$

We assume $k \geq 3$ (the case $k=2$ is similar). In the above construction, we map $\pi=(\pi(1), \sigma, \rho)$ to $\pi^{\prime}=\left(\pi(1), \sigma^{\prime}, \rho^{\prime}\right)$ as in Fig. 1 , where $\sigma^{\prime}=\alpha_{1} \sigma$ and $\rho^{\prime}=\alpha_{2} \rho$. Given $\pi^{\prime}$ and the integers $\left(a_{i}\right), \pi(\mathbf{1})$ is given uniquely as the shortest SAW of $\pi^{\prime}$ starting at $\mathbf{1}$ with span $a_{1}$. Having determined $\pi(\mathbf{1})$, there are no greater than $r+1$ possible choices for the inserted walk $v_{1}:=v\left(\pi_{t}, \alpha_{1} \pi_{n_{2}}\right)$, where $r$ is given after (2.3). For each such choice of $\nu_{1}$, we may identify $\sigma^{\prime}$ as the longest sub-SAW of $\pi^{\prime}$ that is a bridge starting at the second endpoint $z$, say, of $v_{1}$ and ending at a vertex with height $h(z)+a_{2}$. The second inserted SAW $v_{2}:=v\left(\alpha_{1} \pi_{t}, \alpha_{2} \pi_{n_{2}}\right)$, is simply $\alpha_{1} v_{1}$. The SAW $\rho^{\prime}$ is all that remains in $\pi^{\prime}$. In conclusion, for given $\pi^{\prime}$ and $\left(a_{i}\right)$, there are no greater than $r+1$ admissible ways to express $\pi^{\prime}$ in the form $\left(\pi(\mathbf{1}), \sigma^{\prime}, \rho^{\prime}\right)$.

Let $\left(\pi(\mathbf{1}), \sigma^{\prime}, \rho^{\prime}\right)$ be such an admissible representation of $\pi^{\prime}$. Since $\mathcal{H}$ acts by left multiplication, there exists a unique $\alpha_{2} \in \mathcal{H}$ such that $\alpha_{2} \pi_{n}$ equals the final endpoint of $\rho^{\prime}$. It follows that $\rho=\alpha_{2}^{-1} \rho^{\prime}$. By a similar argument, there exists a unique choice for $\sigma$ that corresponds to the given representation.

In conclusion, for given $\pi^{\prime}$ and $\left(a_{i}\right)$, there are no greater than $r+1$ SAWs $\pi$ that give rise to $\pi^{\prime}$. By (6.3)-(6.4),

$$
w\left(H_{m}(v)\right) \leq \begin{cases}\frac{r+1}{\theta_{\min }^{2}} w\left(B_{m+2 s_{i, j}}^{v}\left(a_{1}+a_{2}+a_{3}+2 \delta_{i, j}, a_{4}, \ldots, a_{k}\right)\right) & \text { if } k \geq 3, \\ \frac{r+1}{\theta_{\min }} w\left(B_{m+s_{i, j}}^{v}\left(a_{1}+a_{2}+\delta_{i, j}\right)\right) & \text { if } k=2,\end{cases}
$$


Since $\ell \in \Lambda_{\epsilon}(C, \phi)$ where $\epsilon \in[1,2)$, by Proposition 5.6 the span of any SAW $\pi^{\prime \prime}$ with $w\left(\pi^{\prime \prime}\right)>0$ is no greater than $C \ell\left(\pi^{\prime \prime}\right)^{\epsilon}$. Write $\sum_{a}^{(k, T)}$ for the summation over all finite integer sequences $a_{1}>\cdots>a_{k}>0$ with length $k$ and sum $T$.

By iteration of (6.5), as in [12, Lemma 6.1],

$$
\begin{aligned}
w\left(H_{m}(v)\right) & \leq \sum_{T=1}^{M} \sum_{k=1}^{\sqrt{2 T}} \sum_{a}^{(k, T)} w\left(B_{m}^{v}\left(a_{1}, \ldots, a_{k}\right)\right) \\
& \leq \sum_{T=1}^{M} \sum_{k=1}^{\sqrt{2 T}} \sum_{a}^{(k, T)}\left[\left(\frac{r+1}{\theta_{\min }}\right)^{k-1} \sum_{t=0}^{(k-1) s} w\left(\beta_{m+t}(v)\right)\right]
\end{aligned}
$$

where

$$
M=C(m+s \sqrt{2 M})^{\epsilon} \leq C\left(m+D m^{\epsilon / 2}\right)^{\epsilon},
$$

and $D=D(s)$.

Assume for the moment that $\beta_{\phi, \ell}>1$; a similar argument is valid when $\beta_{\phi, \ell} \leq 1$. By (5.8) and (3.8), there exist constants $D=D\left(\psi, A, a, \theta_{\min }, \theta_{\max }, r, s\right)$, which are continuous in $\psi, A, a, \theta_{\min }, \theta_{\max }$, such that

$$
\begin{aligned}
w\left(H_{m}(v)\right) & \leq \sum_{T=1}^{M} \sum_{k=1}^{\sqrt{2 T}} \sum_{a}^{(k, T)}\left(\frac{r+1}{\theta_{\min }}\right)^{k-1} D k s\left(\beta_{\phi, \ell}\right)^{m+k s+A} \\
& \leq D \sum_{T=1}^{M} \sum_{k=1}^{\sqrt{2 T}} \sum_{a}^{(k, T)}\left(\frac{r+1}{\theta_{\min }}\right)^{\sqrt{2 M}}(s \sqrt{2 M})\left(\beta_{\phi, \ell}\right)^{m+k s+A} \\
& \leq D m^{2 \epsilon} e^{D m^{\epsilon / 2}}\left(\beta_{\phi, \ell}\right)^{m+D m^{\epsilon / 2}}
\end{aligned}
$$

as required.

Proof of Proposition 6.2 We adapt the proof of [12, Prop. 6.6]. In preparation, for $u \in$ $\Gamma$, there exists $\eta_{u} \in \Gamma$ such that $e_{u}:=\left\langle u \eta_{u}, u\right\rangle \in E_{\phi}$ and $h\left(u \eta_{u}\right)<h(u)$. Since $\mathcal{H}$ acts quasi-transitively, we may pick the $\eta_{u}$ such that

$$
J:=\left\lceil\sup \left\{\ell\left(e_{u}\right): u \in \Gamma\right\}\right\rceil
$$

satisfies $J<\infty$.

Let $\pi=\left(\pi_{0}, \pi_{1}, \ldots, \pi_{n}\right)$ be a SAW contributing to $\sigma_{m}(\mathbf{1})$, and let $H=\min \left\{h\left(\pi_{i}\right)\right.$ : $0 \leq i \leq n\}$ and $I=\max \left\{i: h\left(\pi_{i}\right)=H\right\}$. We add the edge $e_{I}$ to $\pi$ to obtain two half-space walks, one from $\pi_{I} \eta_{\pi_{I}}$ to $\pi_{0}$ and the other from $\pi_{I}$ to $\pi_{n}$, with respective $\ell$-lengths $l+\ell\left(e_{I}\right)$ and $L-l$ for some $L \in[m, m+A)$ and $l \in[0, \ell(\pi)]$.

Therefore, $h_{a}:=\max _{v} w\left(H_{a}(v)\right)$ satisfies

$$
w\left(\sigma_{m}\right) \leq \sum h_{a} h_{b},
$$


where the sum is over integers $a, b$ satisfying $l \in[a-J, a+J+A), L-l \in[b, b+A)$. By these constraints,

$$
m-2 A-J \leq a+b \leq m+A+J .
$$

The claim follows from Proposition 6.1 as in [12].

Proof of Theorem 3.10 That $\mu_{\phi, \ell}=\beta_{\phi, \ell}$ follows immediately from Proposition 6.2, and we turn to (3.10). Let $c \geq A$. Since $w\left(\sigma_{m, c}\right) \geq w\left(\beta_{m, c}\right)$, we have

$$
\liminf _{m \rightarrow \infty} w\left(\sigma_{m, c}\right)^{1 / m} \geq \beta_{\phi, \ell},
$$

and hence $w\left(\sigma_{m, c}\right)^{1 / m} \rightarrow \mu_{\phi, \ell}$ as required in part (a) of the theorem. Part (b) holds by (3.9) and the definition (2.2) of the weight of a SAW.

\section{Proof of Theorem 4.1}

We begin with a proposition and then prove Theorem 4.1. The section ends with a proof that, as the truncation of a weight function is progressively removed, the connective constant converges to its original value (see (7.13) and Proposition 7.4).

Proposition 7.1 (a) Let $\left(\phi, \ell_{1}\right),\left(\nu, \ell_{2}\right) \in \Phi \circ \Lambda_{\epsilon}(C, W)$ for some $\epsilon \in[1,2)$ and $C, W>0$. If

$$
\delta:=D_{\text {sup }}\left(\left(\phi, \ell_{1}\right),\left(v, \ell_{2}\right)\right) \text { satisfies } \delta \in(0,1)
$$

then

$$
\mu_{v, \ell_{2}} \leq \begin{cases}B^{\Delta \log \Delta}\left(\mu_{\phi, \ell_{1}}\right)^{\Delta} & \text { if } \mu_{\phi, \ell_{1}}>1 \\ B^{\Delta \log \Delta}\left(\mu_{\phi, \ell_{1}}\right)^{1 / \Delta} & \text { if } \mu_{\phi, \ell_{1}} \leq 1\end{cases}
$$

where

$$
\Delta=\frac{1+\delta}{1-\delta}, \quad B=\exp \left(1 / \ell_{1, \min }\right), \quad \ell_{1, \min }=\min \left\{\ell_{1}(\gamma): \gamma \in \operatorname{supp}(\phi)\right\}
$$

(b) If $v=\phi \in \Phi, \ell_{1}, \ell_{2} \in \Lambda$, and $\delta$ is given by (7.1) and satisfies $\delta \in(0,1)$, then (7.2) holds with $B=1$.

Proof (a) By (7.1), (4.1), and the assumption $\delta<1$, we have that

$$
\begin{aligned}
& \operatorname{supp}(\phi)=\operatorname{supp}(v), \\
& \frac{1}{\Delta}<\frac{\ell_{1}(\gamma)}{\ell_{2}(\gamma)}, \frac{\phi(\gamma)}{v(\gamma)}<\Delta, \quad \gamma \in \operatorname{supp}(\phi) .
\end{aligned}
$$


Let $\pi=\left(\pi_{0}, \pi_{1}, \ldots, \pi_{n}\right)$ be such that $w_{\phi}(\pi)>0$. Then

$$
\begin{aligned}
\frac{w_{v}(\pi)}{w_{\phi}(\pi)} & =\prod_{e \in \pi} \frac{v(e)}{\phi(e)} \leq \prod_{e \in \pi} \Delta \text { by }(7.5) \\
& =\Delta^{n} \leq \exp \left(\frac{\ell_{1}(\pi) \log \Delta}{\ell_{1, \min }}\right) .
\end{aligned}
$$

By (7.5) and (7.6),

$$
w_{\nu}\left(\sigma_{m}\right) \leq \exp \left(\frac{R \log \Delta}{\ell_{1, \min }}\right) \sum_{[L, R)} w_{\phi}(\pi)
$$

where the summation is over SAWs $\pi$ from 1 with $L \leq \ell_{1}(\pi)<R$, and

$$
L=\frac{m}{\Delta}, \quad R=(m+A) \Delta
$$

We take the $m$ th root of (7.7) and let $m \rightarrow \infty$ to obtain (7.2) by (3.10).

(b) When $\phi=v \in \Phi$, the left side of (7.6) equals 1, and the exponential term in (7.7) is replaced by 1 .

Proof of Theorem 4.1 Let $\Gamma$ be virtually $(\mathcal{H}, F)$-indicable. Let $C, W>0, \epsilon \in[1,2)$, and $\left(\phi, \ell_{1}\right) \in \Phi \circ \Lambda_{\epsilon}(C, W)$. We shall assume that $\mu_{\phi, \ell_{1}} \geq 1$ (implying that $W \geq 1$ ); a similar proof is valid otherwise. We shall show continuity at the point $\left(\phi, \ell_{1}\right)$.

Let $\rho>0$, and write

$$
D(\delta):=W\left|B^{\Delta \log \Delta} W^{\Delta-1}-1\right|+W\left|B^{\Delta^{2} \log \Delta} W^{\Delta-1}-1\right|,
$$

where $B$ and $\Delta=\Delta(\delta)$ are given in (7.3). Pick $\delta_{0} \in(0,1)$ such that

$$
D(\delta)<\rho \text { whenever } \delta \in\left(0, \delta_{0}\right) \text {. }
$$

Let $\left(v, \ell_{2}\right) \in \Phi \circ \Lambda_{\epsilon}(C, W)$, write $\delta:=D_{\text {sup }}\left(\left(\phi, \ell_{1}\right),\left(v, \ell_{2}\right)\right)$, and suppose $\delta<\delta_{0}$. We shall prove that

$$
\left|\mu_{\phi, \ell_{1}}-\mu_{v, \ell_{2}}\right|<\rho \text {. }
$$

Note by (7.5) that, since $\delta<\delta_{0}<1$,

$$
\ell_{2, \min } \geq \frac{\ell_{1, \min }}{\Delta}
$$

Assume that $\mu_{v, \ell_{2}} \geq 1$; a similar proof is valid otherwise. By (7.11) and Proposition 7.1(a), 


$$
\begin{aligned}
\mu_{\phi, \ell_{1}}-B^{\Delta \log \Delta}\left(\mu_{\phi, \ell_{1}}\right)^{\Delta} & \leq \mu_{\phi, \ell_{1}}-\mu_{v, \ell_{2}} \\
& \leq B^{\Delta^{2} \log \Delta}\left(\mu_{v, \ell_{2}}\right)^{\Delta}-\mu_{v, \ell_{2}} .
\end{aligned}
$$

Therefore,

$$
\left|\mu_{\phi, \ell_{1}}-\mu_{v, \ell_{2}}\right| \leq D(\delta)<\rho,
$$

by (7.8) and (7.9). Inequality (7.10) is proved.

Let $\phi \in \Phi$. For $\eta>0$, let $\phi^{\eta}: \Gamma \rightarrow[0, \infty)$ be the truncated weight function

$$
\phi^{\eta}(\gamma)= \begin{cases}\phi(\gamma) & \text { if } \phi(\gamma) \geq \eta, \\ 0 & \text { otherwise }\end{cases}
$$

and let $M=M(\phi)=\sup \left\{\eta>0: \phi^{\eta}\right.$ spans $\left.\Gamma\right\}$. By Lemma 5.1, $M>0$.

Definition 7.2 Let $\phi \in \Phi$ and $\ell \in \Lambda$. We say that the pair $(\phi, \ell)$ is continuous at 0 if $\ell(\gamma) \rightarrow \infty$ as $\phi(\gamma) \rightarrow 0$, which is to say that

$$
\forall K>0, \exists \eta>0 \text { such that: } \forall \gamma \text { satisfying } \phi(\gamma) \in(0, \eta) \text {, we have } \ell(\gamma)>K \text {. }
$$

The set of such pairs $(\phi, \ell)$ is denoted $\mathcal{C}$.

Example 7.3 If $\ell \in \Lambda$ is such that $\ell(\gamma)=1 / \phi(\gamma)$ on $\operatorname{supp}(\phi)$, then $(\phi, \ell)$ is continuous at 0. Recall Example 3.6.

Proposition 7.4 Let $\Gamma$ be finitely generated and virtually $(\mathcal{H}, F)$-indicable, and let $(\phi, \ell) \in \Phi \circ \Lambda_{\epsilon}(C)$ for some $C>0$ and $\epsilon \in[1,2)$. Assume in addition that (7.14) holds, in that $(\phi, \ell) \in \mathcal{C}$. Then

$$
\mu_{\phi^{\eta}, \ell} \rightarrow \mu_{\phi, \ell} \text { as } \eta \rightarrow 0
$$

Proof Since $\ell \in \Lambda_{\epsilon}(C, \phi)$ and $\phi^{\eta} \leq \phi$, we have by (3.9) that $\ell \in \Lambda_{\epsilon}\left(C, \phi^{\eta}\right)$ for $\eta<M$. Therefore, Theorem 3.10 may be applied to the pairs $\left(\phi^{\eta}, \ell\right)$.

Let $\zeta \in(0, M)$, so that $\phi^{\zeta}$ spans $\Gamma$. Let $K_{\phi^{\zeta}}$ be the locally finite Cayley graph of $\Gamma$ on the edges $e$ for which $\phi^{\zeta}(e)>0$ (see Lemma 5.1), with corresponding strong graph height function $(h, \mathcal{H})$, and let $\psi, A, a$ be as in (5.3) with $\phi$ replaced by $\phi^{\zeta}$. Working on the graph $K_{\phi^{\zeta}}$ with the weight function $\phi^{\zeta}$, we construct the paths $v_{i, j}$ as before (2.4), and we shall stay with these particular paths in the rest of the proof. Since $\phi^{\eta}$ is constant on these paths for $\eta \leq \zeta$, the values of $\psi, A, a, \theta_{\min }, \theta_{\max }, r, s$ are unchanged for $\eta \leq \zeta$.

Assume $\beta_{\phi, \ell}>1$; a similar proof holds if $\beta_{\phi, \ell} \leq 1$. Take $c=A$ in Theorem 3.10, and let $m \in \mathbb{N}$; later we shall allow $m \rightarrow \infty$. By (7.14), we may choose $\rho=\rho_{m} \in$ $(0, \zeta)$, such that

$$
\text { for any } \gamma \text { satisfying } \phi(\gamma) \in(0, \rho) \text {, we have } \ell(\gamma)>m+A \text {. }
$$


We shall write $w_{\phi}$ since we shall work with more than one weight function. By (7.13)-(7.15),

$$
w_{\phi}\left(\sigma_{m, A}\right)=w_{\phi^{\rho}}\left(\sigma_{m, A}\right) .
$$

Since $\phi^{\rho} \leq \phi$, we have by (3.9) that $\ell \in \Lambda_{\epsilon}\left(C, \phi^{\rho}\right)$. By Proposition 6.2 applied to $\phi^{\rho}$

$$
w_{\phi^{\rho}}\left(\sigma_{m, A}\right) \leq g_{m}\left(\beta_{\phi^{\rho}, \ell}\right)^{m+D m^{\epsilon / 2}},
$$

where $g_{m}=D m^{4 \epsilon} e^{D m^{\epsilon / 2}}$ with the constants $D=D\left(\psi, A, a, \theta_{\min }, \theta_{\max }, r, s\right)$ given as in the proposition. Since $\beta_{\phi^{\rho}, \ell} \leq \mu_{\phi^{\rho}, \ell}$ and (by Theorem 3.10(b)) $\mu_{\nu, \ell}$ is nondecreasing in the weight function $v$, we have by (7.16)-(7.17) that

$$
w_{\phi}\left(\sigma_{m, A}\right) \leq g_{m}\left(\mu_{\phi^{\rho}, \ell}\right)^{m+D m^{\epsilon / 2}} \leq g_{m}\left(\lim _{\eta \rightarrow 0} \mu_{\phi^{\eta}, \ell}\right)^{m+D m^{\epsilon / 2}}
$$

Take $m$ th roots and let $m \rightarrow \infty$, to obtain by (3.10) that

$$
\mu_{\phi, \ell} \leq \lim _{\eta \rightarrow 0} \mu_{\phi^{\eta}, \ell}
$$

and the result follows.

\section{Proof of Theorem 2.5}

We omit this proof since it lies close to those of Theorem 3.10 and [12, Thm 4.3]. Here is a summary of the differences. Theorem 2.5 differs from [12, Thm 4.3] in that edges are weighted, and this difference is handled in very much the same manner as in the proof of Theorem 3.10. On the other hand, Theorem 2.5 differs from Theorem 3.10 in that the underlying graph need not be a Cayley graph. This is handled as in the proof of [12, Thm 4.3], where the unimodularity of the automorphism groups in question is utilized. See also Remark 2.6.

Acknowledgements We thank Agelos Georgakopoulos for enquiring about weighted SAWs on groups. GRG was supported in part by the EPSRC under Grant EP/I03372X/1. ZL was supported in part by the NSF under Grant DMS-1608896. Some of the work was done during a visit by GRG to Keio University, Tokyo. The authors are grateful to two referees for their useful comments.

Open Access This article is distributed under the terms of the Creative Commons Attribution 4.0 International License (http://creativecommons.org/licenses/by/4.0/), which permits unrestricted use, distribution, and reproduction in any medium, provided you give appropriate credit to the original author(s) and the source, provide a link to the Creative Commons license, and indicate if changes were made. 


\section{References}

1. Cassandro, M., Merola, I., Picco, P., Rozikov, U.: One-dimensional Ising models with long range interactions: cluster expansion, phase-separating point. Comm. Math. Phys. 327, 951-991 (2014)

2. Chino, Y.: Sharp transition in self-avoiding walk on random conductors on a tree (2016). arXiv: 1606.08341

3. Chino, Y., Sakai, A.: The quenched critical point for self-avoiding walk on random conductors. J. Stat. Phys. 163, 754-764 (2016)

4. Ding, J., Sly, A.: Distances in critical long range percolation (2013). arXiv:1303.3995

5. Duminil-Copin, H., Smirnov, S.: The connective constant of the honeycomb lattice equals $\sqrt{2+\sqrt{2}}$. Ann. Math. 175, 1653-1665 (2012)

6. Glazman, A.: Connective constant for a weighted self-avoiding walk on $\mathbb{Z}^{2}$. Electron. Commun. Probab. 20, 1-13 (2015)

7. Glazman, A., Manolescu, I.: Self-avoiding walk on $\mathbb{Z}^{2}$ with Yang-Baxter weights: universality of critical fugacity and 2-point function (2017). arXiv:1708.00395

8. Grimmett, G.R.: Percolation, 2nd edn. Springer, Berlin (1999)

9. Grimmett, G.R., Li, Z.: Connective constants and height functions for Cayley graphs. Trans. Amer. Math. Soc. 369, 5961-5980 (2017)

10. Grimmett, G.R., Li, Z.: Self-avoiding walks and amenability. Electron. J. Combin. 24, paper P4.38 (2017)

11. Grimmett, G.R., Li, Z.: Self-avoiding walks and connective constants (2017). arXiv:1704.05884

12. Grimmett, G.R., Li, Z.: Locality of connective constants. Discrete Math. 341, 3483-3497 (2018)

13. Hammersley, J.M.: Percolation processes II. The connective constant. Proc. Cambridge Philos. Soc. 53, 642-645 (1957)

14. Hammersley, J.M., Morton, W.: Poor man's Monte Carlo. J. R. Stat. Soc. B 16, 23-38 (1954)

15. Hammersley, J.M., Welsh, D.J.A.: Further results on the rate of convergence to the connective constant of the hypercubical lattice. Q. J. Math. 13, 108-110 (1962)

16. Hardy, G.H., Ramanujan, S.: Asymptotic formulae for the distribution of integers of various types. Proc. Lond. Math. Soc. 16, 112-132 (1917)

17. Hillman, J.A.: The Algebraic Characterization of Geometric 4-Manifolds. London Mathematical Society Lecture Note Series, vol. 198. Cambridge University Press, Cambridge (1994)

18. Hillman, J.A.: Four-Manifolds, Geometries and Knots. Geometry and Topology Monographs, vol. 5. Mathematical Sciences Publishers, Berkeley (2002). arXiv:math/0212142

19. Lacoin, H.: Existence of a non-averaging regime for the self-avoiding walk on a high-dimensional infinite percolation cluster. J. Stat. Phys. 154, 1461-1482 (2014)

20. Lacoin, H.: Non-coincidence of quenched and annealed connective constants on the supercritical planar percolation cluster. Probab. Theory Related Fields 159, 777-808 (2014)

21. Lindorfer, C.: A general bridge theorem for self-avoiding walks (2019). arXiv:1902.08493

22. Lyons, R., Peres, Y.: Probability on Trees and Networks. Cambridge University Press, Cambridge (2016). http://mypage.iu.edu/ rdlyons/

23. Madras, N., Slade, G.: Self-Avoiding Walks. Birkhäuser, Boston (1993)

24. Trofimov, V.I.: Automorphism groups of graphs as topological groups. Math. Notes 38, 717-720 (1985)

Publisher's Note Springer Nature remains neutral with regard to jurisdictional claims in published maps and institutional affiliations. 